\title{
Randomised double blind trial of oral versus intravenous flecainide for the cardioversion of acute atrial fibrillation
}

\author{
N J Alp, J A Bell, M Shahi
}

\begin{abstract}
Objective-To investigate whether an oral loading dose of flecainide is as safe and effective as intravenous flecainide for the cardioversion of acute atrial fibrillation.

Design-Prospective, randomised, double blind, double placebo study.

Setting-Cardiac care unit of a large district general hospital in the UK.

Patients and methods-79 patients presenting with symptomatic acute atrial fibrillation: patients were given either intravenous flecainide $(n=39)$ or a solution of oral flecainide $(n=40)$, with appropriate placebos. All patients were heparinised during the study.

Primary outcome measures-Safety; mean time to cardioversion; proportion of patients restored to sinus rhythm at two hours and eight hours after treatment. Analysis was by intention to treat.

Results-There were no differences in baseline characteristics between the oral and intravenous groups. Both forms of flecainide were well tolerated, with no adverse clinical events during the study. The mean time to cardioversion was 110 minutes in the oral group and 52 minutes in the intravenous group $(\mathrm{p}=0.002)$. Two hours after treatment, 27 of the 40 patients in the oral group $(68 \%)$ and 25 of the 39 in the intravenous group $(64 \%)$ had reverted to sinus rhythm $(\mathrm{p}=0.74)$. Eight hours after treatment, 30 patients in the oral group (75\%) and 28 in the intravenous group $(72 \%)$ had reverted to sinus rhythm $(\mathrm{p}=0.76)$.

Conclusions-Intravenous flecainide restored sinus rhythm more rapidly than oral flecainide, but at two hours and eight hours after treatment there was no difference in the proportion of patients cardioverted by the two approaches. These results suggest a role for oral loading doses of flecainide in the treatment of acute or symptomatic paroxysmal atrial fibrillation.

(Heart 2000;84:37-40)
\end{abstract}

Keywords: atrial fibrillation; flecainide

Atrial fibrillation is the most common cardiac arrhythmia, with a prevalence of between $2 \%$ and $4 \%$ in the general population over 60 years old, and an increasing incidence with age. ${ }^{1}$ Acute atrial fibrillation (defined as atrial fibrillation of less than 48 hours' duration) is thus a common diagnosis and often requires admission to emergency medical and cardiac care units. Various management strategies are currently employed, but there is agreement that early cardioversion to sinus rhythm (electrical or pharmacological) should be the mainstay of treatment. This provides rapid relief of symptoms, increases the likelihood of long term cardioversion, reduces thromboembolic risk, and may reduce the incidence of further episodes of atrial fibrillation. ${ }^{2}$

Spontaneous reversion of acute atrial fibrillation to sinus rhythm is commonapproximately $20 \%$ of patients return to sinus rhythm within two hours and 50\% within 24 hours, with no specific antiarrhythmic treatment. ${ }^{34}$ Direct current cardioversion is effective in $70-90 \%$ of cases of atrial fibrillation, ${ }^{5}$ but requires a general anaesthetic.

Several randomised controlled trials have evaluated the efficacy of antiarrhythmic drugs for the cardioversion of acute atrial fibrillation, including flecainide, quinidine, procainamide, propafenone, amiodarone, and sotalol. From these studies, intravenous flecainide appears to be one of the most effective drugs, with success rates of between $59 \%$ and $92 \%$ within two hours. ${ }^{4-8}$

Oral routes of flecainide administration have also been evaluated in acute atrial fibrillation. Cardioversion rates of $59-95 \%$ within three to eight hours have been reported in single blind clinical trials. ${ }^{3} 910$

These studies suggest that both intravenous and oral loading doses of flecainide are effective for the cardioversion of acute atrial fibrillation. The present study is the first randomised double blind clinical trial to directly compare the efficacy of these two routes of administration.

\section{Methods}

PATIENTS

The study was performed in the cardiac care unit of a large district hospital in the UK. The local research ethics committee approved the study protocol. Written informed consent was obtained from all study participants. Patients aged over 18 years with symptomatic atrial fibrillation of less than 48 hours' duration and a ventricular rate of more than 100 beats/min were considered eligible for inclusion in the trial. Exclusion criteria were: haemodynamic compromise requiring immediate dc cardioversion; cardiac failure (New York Heart Association functional class III or IV); acute myocar-

\footnotetext{
email: 101323.2347@

compuserve.com

Accepted 15 March 2000

Cardiology
Departmen
Radcliffe Ho
Headley Wa
OX3 9DU, U
N J Alp
Cardiology
Departmen

Berkshire and Battle

RG30 1AG, UK

J A Bell

Correspondence to:
} 


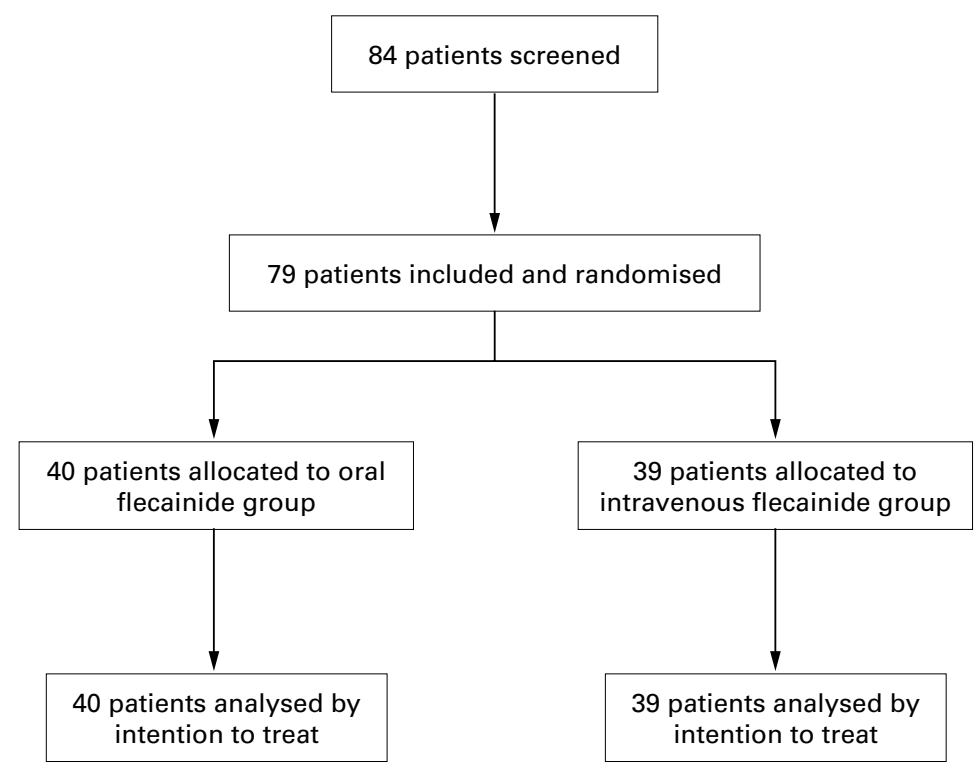

Figure 1 Trial profile.
OUTCOME MEASURES

After enrolment in the study, patients were monitored by ECG continuously, with regular haemodynamic observations as clinically indicated. A 12 lead ECG was recorded at the start of the trial treatment, at two hours and eight hours after treatment, and following any change in cardiac rhythm. To evaluate the effect of flecainide on ventricular depolarisation, the mean 12 lead ECG QRS intervals were measured before and two hours after treatment in both groups.

The primary endpoints of the study were:

- safety, as judged by clinical symptoms haemodynamic status, and arrhythmias;

- the time in minutes to restoration of sinus rhythm;

- whether sinus rhythm was restored at two hours and at eight hours after treatment.

Following the completion of the study period at eight hours after inclusion, trial patients were managed according to the advice of the clinician in charge, in the knowledge that they had received a loading dose of flecainide.

dial infarction at presentation or within the preceding three months; high grade ventricular arrhythmias; pregnancy; severe hepatic failure; severe renal failure; current treatment with flecainide; permanent cardiac pacemaker; and inability to provide informed consent.

TRIAL DESIGN

A prospective randomised double blind, double placebo design was used for the study. Randomisation was done by a computerised random number generator in blocks of 20 . Each consecutive patient was assigned to a study group according to their study entry number, which was kept in an opaque sealed envelope. Patients were randomised to receive either: flecainide $2 \mathrm{mg} / \mathrm{kg}$ (maximum $150 \mathrm{mg}$ ) in $100 \mathrm{ml} \mathrm{5 \%}$ dextrose intravenously over 30 minutes and oral placebo solution; or $100 \mathrm{ml}$ $5 \%$ dextrose infusion over 30 minutes intravenously and oral flecainide $4 \mathrm{mg} / \mathrm{kg}$ (maximum $300 \mathrm{mg}$ ) as a solution (10 mg/ml flecainide). The dose of oral flecainide was twice that of the intravenous dose: this was calculated from pharmacokinetic data on flecainide absorption to obtain similar therapeutic peak plasma concentrations. ${ }^{1112}$ The active oral flecainide solution and the placebo solution (Penn Pharmaceuticals, Tradegar, Gwent, UK) used the same orange flavoured base and tasted identical. The two treatment arms were internally controlled, but we did not use an additional "double placebo" arm on ethical grounds.

One nurse on each shift was responsible for assigning study patients and preparing the treatments, and then took no further part in those patients' care. The admitting physician gave the treatments blinded to their composition. The study randomisation code remained sealed until the planned interim analysis, at which point the trial was terminated. All patients were given weight adjusted intravenous heparin (activated partial thromboplastin time 2.0 to 2.5 times the control) as thromboprophylaxis after inclusion in the study.

\section{STATISTICAL ANALYSIS}

Statistical analysis was on an "intention to treat" basis. We used $\chi^{2}$ tests to compare proportions of patients in sinus rhythm at each end point. Standard parametric tests were used for analysis of time to cardioversion. Parametric tests and $\chi^{2}$ tests were used for comparisons of patient baseline characteristics. Data were analysed using Microsoft Excel 97 software. All reported $\mathrm{p}$ values are two sided.

To calculate the number of patients required to obtain statistical power, a significance level $(\alpha)$ of 0.05 and power $(1-\beta)$ of 0.80 were used. Assuming a treatment efficacy of $75 \%$, we prospectively defined a $15 \%$ difference between the two preparations as not clinically significant (that is, equivalent). For a two sided significance level of 0.05 using a $\chi^{2}$ test to compare proportions, it was calculated that 150 patients would be needed in each arm of the trial. It was intended to perform an interim analysis after 76 patients were enrolled.

\section{Results}

From December 1997 to July 1999, 84 patients were screened, of whom 79 gave consent and were randomised (fig 1). All patients completed the study protocol. At the interim analysis of these 79 patients, a significant difference between the two groups in the time to restoration of sinus rhythm was observed and so the study was terminated. The analysis from these patients is presented.

Baseline characteristics - including age, sex ratio, duration of atrial fibrillation, and risk factor profile-were similar in the two groups (table 1). Nineteen of the 79 patients $(24 \%)$ had a previous history of ischaemic heart disease (chronic stable angina or a previous myocardial infarction) but were free of symptoms at the time of presentation. Twenty four of the patients $(30 \%)$ had a history of hypertension. 
Table 1 Baseline patient characteristics

\begin{tabular}{llll}
\hline Characteristic & $\begin{array}{l}\text { Oral flecainide } \\
(n=40)\end{array}$ & $\begin{array}{l}\text { iv flecainide } \\
(n=39)\end{array}$ & $p$ Value \\
\hline Mean (SD) age (years) & $64.1(14.5)$ & $64.5(15.3)$ & 0.89 \\
Sex (M/F) & $22 / 18$ & $24 / 15$ & 0.55 \\
Duration of AF (h) (mean (range)) & $10.8(1-40)$ & $11.0(1-36)$ & 0.91 \\
History of ischaemic heart disease & $8 / 40$ & $11 / 39$ & 0.40 \\
History of hypertension & $14 / 40$ & $10 / 39$ & 0.38 \\
\hline
\end{tabular}

$\mathrm{AF}$, atrial fibrillation; iv, intravenous.

Table 2 Results of oral and intravenous (iv) flecainide treatment

\begin{tabular}{llll}
\hline Outcome & $\begin{array}{l}\text { Oral flecainide } \\
(n=40)\end{array}$ & $\begin{array}{l}\text { iv flecainide } \\
(n=39)\end{array}$ & $p$ Value \\
\hline Sinus rhythm at two hours (\%) & $27 / 40(68 \%)$ & $25 / 39(64 \%)$ & 0.74 \\
Sinus rhythm at eight hours (\%) & $30 / 40(75 \%)$ & $28 / 39(72 \%)$ & 0.76 \\
Time to cardioversion (min) (mean (SD)) & $110(82.3)$ & $52(54.5)$ & 0.002 \\
\hline
\end{tabular}

SAFETY

Flecainide was well tolerated by either route of administration. Two patients, both in the intravenous group, complained of transient light headedness during the 30 minute flecainide infusion, but there were no haemodynamic changes. One patient in the intravenous group, who had already cardioverted, was noted to have a 30 second episode of asymptomatic ventricular tachycardia (rate 140 beats $/ \mathrm{min}$ ) 60 minutes after treatment, and then reverted to sinus rhythm. This patient was a 66 year old woman with no previous cardiac history, normal electrolytes and renal function, and taking no other drugs. In addition, the cardiac rhythm of one patient in the oral flecainide group 90 minutes after treatment changed from atrial fibrillation to atrial flutter (2:1 atrioventricular block; ventricular rate 100 beats/min) for 20 seconds before cardioverting to sinus rhythm. This patient was a 68 year old woman, also with no previous cardiac history, normal electrolytes and renal function, and taking no other drugs. There were no adverse clinical events during the study period.

Flecainide treatment caused a small but significant prolongation of the QRS interval. In the oral group the mean QRS interval increased from $74 \mathrm{~ms}$ to $85 \mathrm{~ms}$ two hours after treatment $(p<0.001)$. In the intravenous group the mean QRS interval increased from $75 \mathrm{~ms}$ to $81 \mathrm{~ms}$ two hours after treatment $(p=0.03)$. There was no significant difference between the oral $(85 \mathrm{~ms})$ and intravenous (81 ms) groups in the mean QRS interval prolongation at two hours $(\mathrm{p}=0.08)$.

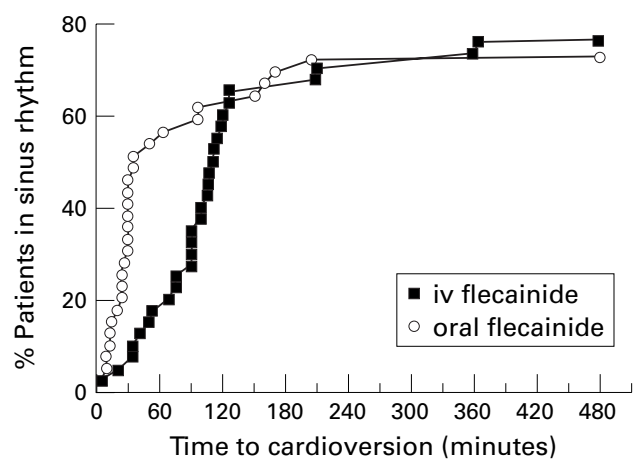

Figure 2 Cumulative cardioversion rates for oral and intravenous flecainide.
CLINICAL ENDPOINTS

The mean time to cardioversion was 110 minutes in the oral group and 52 minutes in the intravenous group $(p=0.002)$ (table 2). A graph of the cumulative cardioversion rates shows the clinical efficacy of the two treatments (fig 2).

Two hours after treatment, 27 of the 40 patients given oral flecainide $(68 \%)$ and 25 of the 39 given intravenous flecainide $(64 \%)$ had reverted to sinus rhythm $(p=0.74)$. Eight hours after treatment 30 patients $(75 \%)$ given oral flecainide and $28(72 \%)$ given intravenous flecainide had reverted to sinus rhythm $(p=0.76)$ (table 2). None of the patients had any further episodes of atrial fibrillation during the study period.

\section{Discussion}

This is the first double blind randomised trial to compare intravenous and oral routes of flecainide loading for cardioversion of acute atrial fibrillation. Intravenous flecainide restored sinus rhythm more quickly than oral flecainide (52 $v 110$ minutes). However, there was no significant difference between the two routes of treatment in the proportions of patients cardioverted by two hours and eight hours.

Both routes of flecainide administration were well tolerated and safe in terms of adverse arrhythmias and clinical events. It is of interest that the one patient who had a 30 second episode of asymptomatic ventricular tachycardia following intravenous flecainide had no evidence of structural heart disease, had normal renal function, and was taking no other drugs. We used only the history and examination, a resting 12 lead ECG, and a chest radiograph to evaluate the patients' suitability for treatment with flecainide. We did not include echocardiography for the assessment of left ventricular function or structural heart disease, as this may not be available in the potential settings where oral flecainide treatment could be of particular value. Patients with acute or recent myocardial infarction, or clinical evidence of cardiac failure, were excluded from our study because of concerns raised by the cardiac arrhythmia suppression trial (CAST). ${ }^{13}$ The CAST trial reported an excess of cardiac and sudden arrhythmic death among patients treated with long term flecainide following acute myocardial infarction. However, the results from CAST may not relate to our study, as high risk patients were excluded and patients received only a single loading dose of flecainide.

The time to cardioversion was approximately 60 minutes faster with intravenous than with oral flecainide. However, some of this time may be offset by the ease of oral administration, compared with the delay involved in inserting an intravenous cannula and preparing an infusion pump.

There was no significant difference in the proportion of patients cardioverted by two hours and eight hours after treatment. This may have implications for the future treatment of acute atrial fibrillation. For example, patients on general hospital wards who develop 
acute atrial fibrillation could be given an oral loading dose of flecainide before being transferred to an acute monitoring ward. In addition, an oral loading dose of flecainide may be useful for patients with occasional symptomatic paroxysmal atrial fibrillation, rather than using chronic antiarrhythmic treatment.

Our results are similar to those from other studies of oral and intravenous flecainide. In one single blind, placebo controlled study of patients with acute atrial fibrillation, an oral loading dose of $300 \mathrm{mg}$ flecainide (as a tablet) was compared to intravenous amiodarone. ${ }^{3}$ Ninety five per cent of the patients in the flecainide group cardioverted within eight hours (the mean (SD) time to cardioversion was 190 (147) minutes). By comparison, 37\% of patients in the amiodarone group and $48 \%$ of patients in the placebo group cardioverted within the same time period. In a further placebo controlled, single blind study of 181 patients, oral flecainide (300 mg given as a tablet) was compared with oral propafenone. ${ }^{9}$ By three hours after treatment, $59 \%$ of patients given flecainide cardioverted, compared with $51 \%$ given propafenone and $18 \%$ given placebo. By eight hours after treatment the numbers of patients cardioverted had increased to $78 \%, 72 \%$, and $39 \%$, respectively. Although we did not include a double placebo group in our study, one placebo controlled trial of intravenous flecainide and amiodarone with similar patient characteristics showed the spontaneous cardioversion rate at two hours to be $22 \% .{ }^{4}$ In a previous non-blinded trial of oral versus intravenous flecainide, 10 of 14 patients given oral flecainide as a tablet cardioverted within five hours compared with 10 of 13 patients given intravenous treatment. ${ }^{10}$ However, the smaller number of patients in the study and the non-blinded design limits interpretation of these results.

\section{LIMITATIONS}

Our study has some limitations. The trial was terminated early because a significant difference in the time to cardioversion had emerged between the two groups. Although there was no significant difference in the proportions of patients cardioverted at two hours and eight hours, the trial was underpowered at this stage to exclude a true difference between the two treatments. However, the profile of the cumulative cardioversion plots suggests that a large difference in efficacy between the treatments at two and eight hours would be unlikely. We used an oral solution of flecainide rather than tablets in our study to obtain an accurate weight adjusted dosing regimen (maximum dose $300 \mathrm{mg}$ ). It is likely that a flecainide tablet would be absorbed slightly more slowly than the solution, but the results of the studies above suggest that oral loading with up to $300 \mathrm{mg}$ of flecainide tablets also leads to cardioversion in $75-90 \%$ of patients within eight hours. We did not perform echocardiography in the assessment of patients before inclusion in the study. We may therefore have missed patients with subclinical left ventricular impairment, structural heart disease, or intracardiac thrombus. However, no adverse events were recorded, and the purely clinical exclusion criteria we used permit application of the results to a wider patient population, who may benefit from oral flecainide treatment in settings where echocardiography screening is not available.

\section{CONCLUSIONS}

We have shown that oral flecainide loading is a safe and effective alternative to intravenous flecainide for the cardioversion of acute atrial fibrillation. These results have implications for the treatment of acute and paroxysmal atrial fibrillation, in that a single oral flecainide loading dose could be used to promote early cardioversion, or as an alternative to chronic antiarrhythmic treatment.

We are grateful to our nursing and medical colleagues in the cardiac care unit for their help with recruitment of patients for the study.

1 Petersen P, Godtfredsen J. Atrial fibrillation: a review of course and prognosis. Acta Med Scand 1984;306:1018-22. 2 Wijffels MCEF, Kirchhof CJHJ, Dorland R, et al. Atrial chrillation begets atrial fibrillation: a study in awake 68.

3 Capucci A, Lenzi T, Boriani G, et al. Effectiveness of loading oral flecainide for converting recent-onset atrial fibrillation to sinus rhythm in patients without organic heart disease or with only systemic hypertension. Am $f$ Cardiol ease or with only

4 Donovan KD, Power BM, Hockings BE, et al. Intravenous flecainide versus amiodarone for recent-onset atrial fibrillation. Am f Cardiol 1995;75:693-7.

5 Van Gelder IC, Crijns HJ, Van Gilst WH, et al. Prediction of uneventful cardioversion and maintenance of sinus rhythm from direct-current cardioversion of chronic atrial fibrillation and flutter. Am $\mathcal{F}$ Cardiol 1991;68:41-6.

6 Madrid AH, Moro C, Marin-Huerta E, et al. Comparison of flecainide and procainamide in cardioversion of atrial fibrillation. Eur Heart f 1993;14:1127-31.

7 Kingma JH, Suttorp MJ. Acute pharmacological conversion of atrial fibrillation and flutter: the role of flecainide, propafenone and verapamil. Am $\mathcal{f}$ Cardiol 1992;70:56$60 A$.

8 Suttorp MJ, Kingma JH, Lie-A-Huen L, et al. Intravenous flecainide versus verapamil for acute conversion of paroxysmal atrial fibrillation or flutter to sinus rhythm. Am f Cardiol 1989;63:693-6.

9 Capucci A, Boriani G, Botto GL, et al. Conversion of recent-onset atrial fibrillation by a single oral loading dose of propafenone or flecainide. Am f Cardiol 1994;74:503-5.

10 Crijns HJ, van Wijk LM, van Gilst WH, et al. Acute conversion of atrial fibrillation to sinus rhythm: clinical efficacy of flecainide acetate. Comparison of two regimens. Eur Heart f 1988;9:634-8

11 Lie-A-Huen L, Proost JH, Kingma JH, et al. Absorption kinetics of oral and rectal flecainide in healthy subjects. Eur 7 Clin Pharmacol 1990;38:595-8.

12 Hage K, Buhl K, Fischer C, et al. Estimation of the absolute bioavailability of flecainide using stable isotope technique. Eur f Clin Pharmacol 1995;48:51-5.

13 Echt DS, Liebson PR, Mitchell LB, et al. Mortality and morbidity in patients receiving encainide, flecainide or placebo. The Cardiac Arrhythmia Suppression Trial. $\mathcal{F} \mathrm{Am}$ Coll Cardiol 1991;18:1434-8. 